\title{
The role of primary tumour sidedness, EGFR gene copy number and EGFR promoter methylation in RAS/BRAF wild-type colorectal cancer patients receiving irinotecan/cetuximab
}

Laura Demurtas ${ }^{1}$, Marco Puzzoni ${ }^{1}$, Riccardo Giampieri ${ }^{2}$, Pina Ziranu ${ }^{1}$, Valeria Pusceddu ${ }^{1}$, Alessandra Mandolesi ${ }^{3}$, Chiara Cremolini ${ }^{4}$, Gianluca Masi ${ }^{4}$, Fabio Gelsomino ${ }^{5}$, Carlotta Antoniotti ${ }^{4}$, Cristian Loretelli ${ }^{2}$, Fausto Meriggi ${ }^{6}$, Alberto Zaniboni ${ }^{6}$, Alfredo Falcone ${ }^{4}$, Stefano Cascinu ${ }^{5}$ and Mario Scartozzi ${ }^{\star}, 1$

${ }^{1}$ Departments of Medical Oncology, University of Cagliari, University Hospital 'Duilio Casula' S.S. 554, Km 4,500 Bivio per Sestu, Monserrato, Cagliari 09042, Italy; ${ }^{2}$ Departments of Medical Oncology, Polytechnic University of the Marche Region, University Hospital, Via Conca 71, Ancona, 60126, Italy; ${ }^{3}$ Departments of Institute of Pathology, AO Ospedali Riuniti-UNIVPM, Via Conca 71, Ancona, 60126, Italy; ${ }^{4}$ Polo Oncologico, Azienda Ospedaliero-Universitaria Pisana, via Roma 67, Pisa, 56126, Italy; ${ }^{5}$ Modena Cancer Centre, Department of Oncology/Haematology, University of Modena and Reggio Emilia, via del Pozzo, Modena, 41124, Italy and ${ }^{6}$ Medical Oncology Unit, Fondazione Poliambulanza, via Bissolati 57, Brescia, 25124, Italy

Background: The data from randomised trials suggested that primary tumour sidedness could represent a prognostic and predictive factor in colorectal cancer (CRC) patients, particularly during treatment with anti-epidermal growth factor receptor (EGFR) therapy. However, an in-deep molecular selection might overcome the predictive role of primary tumour location in this setting.

Methods: We conducted a retrospective analysis in which tumour samples from RAS/BRAF wild-type (WT) metastatic CRC patients treated with second-third-line irinotecan/cetuximab were analysed for EGFR gene copy number (GCN) and promoter methylation. Study objective was to evaluate the correlation of tumour sidedness, EGFR promoter methylation and EGFR GCN with clinical outcome. Median follow-up duration was 14.3 months.

Results: Eighty-eight patients were included in the study, 27.3\% had right-sided CRC, 72.7\% had left-sided CRC; 36.4\% had EGFR $\mathrm{GCN}<2.12$ tumour, 63.6\% had EGFR GCN $\geqslant 2.12$ tumour; 50\% had EGFR promoter-methylated tumour. Right-sided colorectal cancer (RSCRC) were associated with reduced overall response rate (ORR) (4.2\% for RSCRC vs $35.9 \%$ for left sided colorectal cancer (LSCRC), $P=0.0030)$, shorter progression-free survival (PFS) (3.0 vs 6.75 months, $P<0.0001)$ and shorter overall survival (OS) (8 vs 13.6 months, $P<0.0001$ ). EGFR GCN $<2.12$ tumours were associated with reduced ORR (6.2\% for EGFR GCN $<2.12$ vs $39.3 \%$ for EGFR GCN $\geqslant 2.12$ tumours, $P=0.0009$ ), shorter PFS (3.5 vs 6.5 months, $P=0.0006$ ) and shorter OS (8.5 vs 14.0 months, $P<0.0001)$. Epidermal growth factor receptor-methylated tumours were associated with reduced ORR $(9.1 \%$ for methylated vs 45.5\% for unmethylated, $P=0.0001$ ), shorter PFS (3 vs 7.67 months, $P<0.0001$ ) and shorter OS (8 vs 17 months, $P<0.0001$ ). At multivariate analysis, EGFR GCN and EGFR promoter methylation maintained their independent role for ORR (respectively $P=0.0082$ and 0.0025 ), PFS (respectively $P=0.0048$ and $<0.0001$ ) and $O S$ (respectively $P=0.0001$ and $<0.0001$ ).

Conclusions: In our study, an accurate molecular selection based on an all RAS and BRAF analysis along with EGFR GCN and EGFR promoter methylation status seems to be more relevant than primary tumour sidedness in the prediction of clinical outcome during cetuximab/irinotecan therapy. However, these data need to be validated with future prospective and translational studies.

*Correspondence: Professor M Scartozzi; E-mail: marioscartozzi@gmail.com

Received 27 March 2017; revised 3 May 2017; accepted 26 May 2017; published online 20 June 2017

(C) 2017 Cancer Research UK. All rights reserved 0007-0920/17 
Although the global therapeutic strategy has considerably improved in the past few years, colorectal cancer (CRC) still represents a leading cause of death worldwide (Ferlay et al, 2013, 2014). The fast-growing knowledge of molecular mechanisms underlying colorectal tumours led in fact to a corresponding development of effective molecular targeted treatments able to significantly improve clinical outcome for patients presenting with metastatic disease from the original 6-10 months median survival to the current 30 months and more (Van Cutsem et al, 2016).

We now know that metastatic CRC is a biologically heterogeneous disease and primary tumours originating from different areas of the colon have critical molecular differences that have been suggested to translate into just as many critical differences under the clinical point of view. In particular, different data sets indicated that right-sided colorectal cancers (RSCRC) patients experienced a worse clinical outcome than left-sided colorectal cancer (LSCRC) patients with otherwise comparable clinical characteristics (Loupakis et al, 2015; Moretto et al, 2016; Tejpar et al, 2016; Venook et al, 2016).

Interestingly, findings from subset analyses of phase 3 trials also indicated a possible lack of efficacy for the use of anti-epidermal growth factor receptor (EGFR) monoclonal antibodies (that is, cetuximab and panitumumab) in metastatic colorectal cancer patients with RAS wild-type (WT) RSCRC. On the contrary, RAS WT LSCRC patients apparently showed to derive an increasingly significant clinical benefit from such a treatment approach. Taken together, these observations implied that along with a well-established prognostic role, primary tumour location could also represent a relevant predictive factor in colorectal cancer patients receiving treatment directed against the EGFR.

The molecular pathways leading to left- and right-sided primary CRC are sensibly different and could ultimately explain the distinct biological and clinical traits of these tumours.

Right-sided colorectal cancer are more often hyper-mutated, present microsatellite instability and CpG-islands methylation and BRAF mutations. On the other hand, LSCRC have more likely chromosomal instability, EGFR and HER2-neu amplifications, epiregulin and amphiregulin (EREG and AREG) high gene expression levels (Distler and Holt, 1997; Iacopetta, 2002; Hutchins et al, 2011; Tran et al, 2011; Missiaglia et al, 2014; Loupakis et al, 2015; Choi et al, 2016; Moretto et al, 2016; Tejpar et al, 2016; Venook et al, 2016; Lee et al, 2016a). Patients with tumors that have high gene expression levels of EREG and AREG, are more likely to have disease control on cetuximab treatment (Khambata-Ford et al, 2007).

Recently, Lee et al indicated that, in K-RAS wild-type metastatic CRC patients receiving anti-EGFR therapy, the molecular characteristics that are considered typical of RSCRC more frequently overlapped with the consensus molecular subtypes (CMS) of colorectal cancer type 1 (MSI immune), whereas CMS type 3 and 4 were recurrent in LSCRC (Guinney et al, 2015; Lee et al, 2016b).

The authors also found a correlation between the different molecular characteristics investigated and survival results, thus confirming a consistent link between molecular features and clinical outcome. Overall, these findings suggested that besides primary tumour location, the biological profile might be actually more relevant in defining the clinical behaviour of colorectal cancer.

We could then speculate that the more accurate the biological selection is the less relevant primary tumour location would be in the prediction of clinical outcome during treatment with monoclonal antibodies directed against the EGFR.

On this basis, we analysed the prognostic and predictive role of CRC primary tumour location, EGFR GCN and EGFR promoter methylation in RAS and BRAF wild-type mCRC patients treated with second- third line cetuximab/irinotecan therapy.

\section{PATIENTS AND METHODS}

We retrospectively collected primary tumour samples from RAS/ BRAF WT metastatic CRC patients treated with second-third-line irinotecan/cetuximab and analysed them for EGFR GCN and promoter methylation.

Patients selection. Patients with histologically proven, RAS and BRAF wild-type, metastatic colorectal cancer receiving a combination of cetuximab and irinotecan after at least one line of previous chemotherapy were eligible for our analysis.

All patients received cetuximab at an initial dose of $400 \mathrm{mg} \mathrm{m}^{-2}$ followed by weekly infusions of $250 \mathrm{mg} \mathrm{m}^{-2}$. Irinotecan was administered at a dose of $180 \mathrm{mg} \mathrm{m}^{-2}$ every 2 weeks, either alone or in combination with 5-fluorouracil and leucovorin. So, they received modified FOLFIRI (irinotecan $180 \mathrm{mg} \mathrm{m}^{-2} \mathrm{~d} 1$, $5 \mathrm{FU}$ bolus $400 \mathrm{mg} \mathrm{m}^{-2} \mathrm{~d} 1,5 \mathrm{FU} 2400 \mathrm{mg} \mathrm{m}^{-2}$ continuous infusion for $46 \mathrm{~h}$ ) plus cetuximab or irinotecan plus cetuximab.

To be eligible, patients must also have previously received an irinotecan-based chemotherapy regimen for at least 6 weeks and must have presented progression of disease during receipt of this regimen or within 3 months thereafter. All patients received firstline chemotherapy (oxaliplatin-based or irinotecan-based) in combination with bevacizumab. For patients receiving third-line cetuximab, second-line treatment consisted of modified FOLFIRI in all cases.

Tumour response was evaluated every 8 weeks by clinicians' assessment and according to the Response Evaluation Criteria in Solid Tumours (RECIST v1.1).

Median follow up duration was 14.3 months.

Epidermal growth factor receptor promoter methylation and EGFR GCN were analysed on formalin-fixed and paraffinembedded tumour samples for all patients included. For study purposes, right-sided and left-sided colorectal primary tumours were defined as proximal or distal to the splenic flexure. This study was approved by the Institutional Review Board.

Mutational analysis. Evaluation for RAS and B-raf status was conducted for all patients included in the analysis by standard pyrosequencing technique: all evaluations have been performed by PyroMark Q96 ID system (Qiagen, Germany) or by Pyromark ID system (Biotage $A B$, Sweden).

The kits 'Anti-EGFR MoAb response', respectively for K-ras, $\mathrm{N}$-ras and B-raf status, allow to identify the most common and prevalent mutations of K-ras, N-ras and B-raf gene.

The kit for K-ras evaluates hot-spots mutations in codons 12, $13,59,61,117$ and 146 of $K$-ras gene (predominantly G12D, G12A, G12V, G12S, G12R, G12C, G13D, A59T, Q61H, K117N, A146T).

The kit for N-ras evaluates hot-spots mutations in codons 12, $13,59,61,117$ and 146 of $N$-ras gene (predominantly G12S, G12D, G13R, G13D, A59T, Q61K, Q61L, Q61R, K117N, A146T).

The kit for B-raf evaluates hot-spots mutations in codons 15 and 11 of B-raf gene (predominantly V600E, V600K, V600M, T599M, K601E, G469V-A-E and G464V-E).

After tumour DNA extraction and amplification (through Rotor-Gene Q, Qiagen, Germany), genotyping and allele frequencing is determined by standard pyrosequencing technique: the detection of bioluminescence caused by the nucleotide annealing to the sequence and the relative intensity of the luminescence produced is directly proportional to the number of annealed nucleotides caused by the reaction of the DNA polymerase, that starting from the primers used, do appear at the end of the amplification.

Variant allele frequencies (VAF) for the different analyses, by manufacturer's description are as follows:

K-ras codon 12: $10 \%$

K-ras codon 13: $8 \%$ 
K-ras codon 59 pos1: A $12-15 \%$, T 4-7\%, C $8-12 \%$

K-ras codon 61 pos3: C 7-10\%, T $8-12 \%$

K-ras codon 59 pos2: G $12-15 \%$, T 4-7\%, A12-15\%

K-ras codon 61 pos2: C $12-15 \%$, G 4-7\%, T 4-7\%

K-ras codon 61 pos 1: G 8-12\%, A 4-7\%

K-ras codon 117 pos3: G $12-15 \%$, A8-12\%

K-ras codon 117 pos1: G 8-12\%, C $8-12 \%$

K-ras codon 117 pos2: A 8-12\%, C 8-12\%, G 8-12\%

K-ras codon 146 pos 1: G $8-12 \%$, A $12-15 \%$, T $8-12 \%$

K-ras codon 146 pos 2: T 12-15\%, A 12-15\%, C 12-15\%

N-ras codon 12 pos1: T 3-5\%, A 5-7\%, C 3-5\%

$\mathrm{N}$-ras codon 13 pos1: T 7-10\%, A 4-6\%, C 3-5\%

N-ras codon 12 pos2: T 6-9\%, A 6-8\%, C 3-5\%

N-ras codon 13 pos2: T 7-10\%, A 4-6\%, C 4-6\%

$\mathrm{N}$-ras codon 58 pos 1: G 3-5\%, T $8-10 \%$, C $3-5 \%$

N-ras codon 59 pos 1: A $9-11 \%$, T 3-5\%, C $8-10 \%$

N-ras codon 61 pos 1: A $4-7 \%$, G 3-5\%

$\mathrm{N}$-ras codon 58 pos 2: T $8-10 \%$

$\mathrm{N}$-ras codon 59 pos 2: $3-5 \%$

N-ras codon 61 pos 2: G 3-5\%, T 8-10\%, C 4-6\%

$\mathrm{N}$-ras codon 61 pos 3: T 4-6\%, C 7-9\%

N-ras codon 117 pos 1/3: 4-6\%

N-ras codon 117 pos 2: $6-8 \%$

$\mathrm{N}$-ras codon 146 pos 1: $5-7 \%$

N-ras codon 146 pos 2: $8-10 \%$

B-raf codon 600: E 3-5\% K 8-10\% M 8-10\%

B-raf codon 599: I 8-10\%

B-raf codon 601: E 8-10\%

B-raf exon 11 codon 469 and 464: 8-10\%.

EGFR promoter methylation. $\mathrm{CpG}$ island methylation is an epigenetic mechanism of gene silencing more frequently observed in right- than left-sided tumors and the methylation of the EGFR promoter may be responsible for the loss of EGFR expression.

Analysis of EGFR promoter methylation was performed following a DNA extraction protocol from paraffin-embedded tissue and a methylation-specific PCR (MSP) as previously described (Scartozzi et al, 2011). Analysis of EGFR promoter methylation was performed following a DNA extraction protocol from paraffin-embedded tissue and a methylation-specific PCR (MSP). The tumour samples were processed according to the QIAamp DNA mini Tissue Protocol, using QIAamp DNA Mini Kit (Qiagen GmbH, Hilden, Germany). Before PCR amplification, the DNA extract was treated with sodium bisulphite as described in the handbook of the 'EpiTect Bisulfite Kit' (Qiagen GmbH). Bisulphite modification of DNA to convert all unmethylated cytosines to uracil and then to thymidine during the subsequent PCR step while leaving the methylated cytosines unaffected was performed as described by Herman et al, 1996. For PCR amplification, two sets of primers were designed from nt -130 to -300 (relative to ATG) in the $5^{\prime}$-untranslated region of the human EGFR promoter.

The primer sequences used were $5^{\prime}$-TGTTTTGTTTTTTTGTG TTTTGGTTTGTGT- $3^{\prime}$ (sense) and $5^{\prime}$-CATCCAATCTAAACAACAACAACCACCA-3' (antisense) for unmethylated DNA and $5^{\prime}$ $5^{\prime}$-TGTTTTTTCGCGTTTCGGTTCGCGC-3' (sense) and $5^{\prime}$-C GTCTAAACGACGACGACCGCCG-3'(antisense) for methylated DNA, both of which amplify $\sim 150 \mathrm{bp}$ products (Nagothu et al, 2004). The PCR mixture contained $\sim 1 \AA$ PCR buffer, minus Mg; $0.2 \mathrm{~m}$ dNTP mixture (each); $1.5 \mathrm{~m} \mathrm{MgCl} ; 0.2 \mu$ primer mix (each); 1.0 unit platinum Taq DNA polymerase (Invitrogen, Carlsbad, CA, USA); and bisulphite-modified DNA (of $1 \mathrm{ng}-2 \mu \mathrm{g}$ ) in a final volume of $50 \mu$ l. Controls without DNA were performed for each set of PCRs. Each PCR product $(30 \mu \mathrm{l})$ was directly visualised on $10 \%$ acrylamide gels. The gel was stained with ethidium bromide and photographed under UV illumination. An enzymatically methylated human male genomic DNA (CpGenome Universal
Methylated DNA CHEMICON International) was used as a methylation-positive control for gene methylation studies and was processed as above mentioned.

EGFR GCN. Epidermal growth factor receptor GCN study was performed with CISH analysis (chromogenic in situ hybridisation) performed according to manufacturer's instructions (Zymed Laboratories Inc., South San Francisco, CA, USA) as previously described. The cutoff point with the highest sensitivity and specificity for estimating CISH EGFR GCN was set at 2.12, according to our previous findings.

We previously performed a receiver operating characteristics (ROC) analysis based on mean CISH EGFR gene copy number with response to cetuximab therapy as end point. In that model sensitivity was $51.5 \%$ (95\% CI: 33.6-69.2) and specificity was $100 \%$ (95\% CI: 69-100). AUC was 0.77, $P=0.002$ (Scartozzi et al, 2009; Scartozzi et al, 2012).

Statistical analysis. Statistical analysis was performed with the MedCalc Statistical Software version 14.10.2 (MedCalc Software bvba, Ostend, Belgium; http://www.medcalc.org; 2014).

The association between categorical variables was estimated by Fisher exact test for categorical binomial variables or by $\chi^{2}$ test for all other instances. Survival probability over time was estimated by the Kaplan-Meier method. Significant differences in the probability of survival between the strata were evaluated by log-rank test. Cox's multiple regression analysis was used to assess the role of tumour sidedness, EGFR promoter methylation and EGFR GCN as prognostic factors adjusted for those variables resulted significant at univariate analysis. The Holm-Sidak correction 18 was used to adjust the values for multiple comparisons. Other tested variables included gender (male $v s$ female), median age $(<70 v s \geqslant 70$ years), ECOG performance status $(0 v s \geqslant 1)$, previous lines for metastatic disease ( 1 vs 2).

For statistical analysis, overall survival (OS) was defined as the time interval between the date of beginning of cetuximab/ irinotecan treatment and death or last follow-up visit for patients who were lost at follow-up, whereas progression-free survival (PFS) was defined as the interval between the date of beginning of cetuximab/irinotecan treatment to death, first sign of clinical progression or last follow-up visit for patients who were lost at follow-up.

\section{RESULTS}

A total of eighty-eight RAS/BRAF wild type metastatic colorectal cancer patients treated with second-third-line irinotecan/cetuximab therapy were included in our analysis. Twenty-four patients (27\%) had right sided colorectal cancer and 64 patients $(73 \%)$ had left-sided colorectal cancer. EGFR GCN $<2.12$ was present in 32 colorectal tumours (36\%), whereas the remaining $56(64 \%)$ had EGFR GCN $\geq 2.12$. Forty-four patients (50\%) showed EGFR gene promoter methylated tumours. EGFR promoter methylation and EGFR amplification are not mutually exclusive. Twenty-five patients $(28.4 \%)$ presented both parameters.

Overall clinical and pathological patients' characteristics were well balanced across different groups and are summarised in Table 1.

In the whole-group median OS (mOS) was 10 months (95\% CI: 9.0 to 13.6 months), median PFS (mPFS) was 4 months (95\% CI 3.6 to 6.2 ) and overall response rate (ORR) was $27.3 \%$.

RSCC $v s$ LSCC. Right-sided colon cancer were associated with reduced ORR (4.2\% for RSCC vs $35.9 \%$ for LSCC, $P=0.003$ ); mPFS was 3 months (95\% CI 2,9 to 3,5) for RSCC vs 6.75 months (95\% CI: 5.8 to 7.7 ) for LSCC (HR: $2.7, P<0.0001$ ) (Figure 1A). 
RSCC patients also showd a dcreased OS: mOS was 8 months (95\% CI: 4.0 to 8.9 ) vs 13.6 months (95\% CI: 10.0 to 17.5 ) for RSCC vs LSCC (HR: 2.83, $P<0.0001$ ) (Figure 1B).

Epidermal growth factor receptor gene promoter methylation was more frequent in RSCC vs LSCC (respectively 95.8\% vs 32.8\%, $P<0.0001)$. On the contrary EGFR GCN $\geq 2.12$ was less frequently observed in RSCRC vs LSCRC (respectively $16.7 \%$ vs $81.2 \%$, $P<0.0001)$

EGFR GCN. Epidermal growth factor receptor $\mathrm{GCN}<2.12$ was associated with a reduced ORR (6.2\% for patients showing EGFR $\mathrm{GCN}<2.12$ vs $39.3 \%$ for those showing EGFR GCN $\geqslant 2.12$, $P=0.0009$ ) asnd with a shorter PFS and OS. PFS was 3.5 months (95\% CI: 3.0 to 4.5 ) vs 6.5 months (95\% CI: 3.9 to 8.0 ) HR: 1.98 , $P=0.0006$ (Figure 2A) in patients with colorectal tumours showing EGFR GCN respectively $<2.12$ or $\geqslant 2.12$. Overall survival was 8.5 months (95\%CI: 6.0-9.8) vs 14 months (95\% CI: $11.0-18.0$ ) for patients with colorectal tumours showing EGFR GCN $<2.12$ v EGFR GCN $\geqslant 2.12$ (HR: 2.59, $P<0.0001$ ) (Figure $2 B$ ).

EGFR methylation. Patients with EGFR-methylated tumours vs those with unmethylated tumours showed a reduced ORR $(9.1 \%$ for methylated $v s 45.5 \%$ for unmethylated tumours, $P=0.0001$ ). Median PFS was 3 months (95\% CI: 2.5 to 3.2) vs 7.67 months (95\% CI: 6.9 to 8.1 ), HR: $3.23, P<0.0001$ (Figure $3 \mathrm{~A}$ ), and $\mathrm{mOS}$ was 8 months (95\% CI: 4.0 to 8.9) vs 17 months (95\% CI: $13.6-$ 19.0) for patients with methylated $v s$ unmethylated colorectal tumours, respectively (HR: 3.57, $P<0.0001$ ) (Figure 3B). All results have been also summarised in Table 2 .

EGFR GCN $\geq 2.12$ and EGFR unmethylated tumours. Among patients with EGFR GCN $\geqslant 2.12$ tumours, those with unmethylated tumours had a better survival: mOS was 18 months (95\% CI: 1719.5) for unmethylated vs 8.4 (95\% CI: 6-9.8) for methylated tumours, HR 0.27, $P<0.0001$; mPFS was 8 months for unmethylated (95\% CI: 7.6-9) vs 2.9 months for methylated tumours (95\% CI: 2.0 to 4.0$)$, HR: $0.31, P<0.0001$. They showed also better ORR: it was $58 \%$ for unmethylated $v s 16 \%$ for methylated tumours, $P=0.001$.

Multivariate analysis. At multivariate analysis, EGFR GCN and EGFR promoter methylation maintained their independent role for ORR (respectively $P=0.0082$ and $P=0.0025$ ), PFS (respectively $\operatorname{Exp}(\mathrm{b}): 0.408,95 \%$ CI: $0.2-0.8, P=0.0048$ and $\operatorname{Exp}(b): 4.52,95 \%$ CI: $2.5-8.1, P<0.0001)$ and OS (respectively $\operatorname{Exp}(\mathrm{b}): 0.23,95 \% \mathrm{CI}$ : $0.1-0.5, P=0.0001$ and $\operatorname{Exp}(\mathrm{b}): 5.58 .95 \% \mathrm{CI}: 2.8-11.1, P<0.0001$ ).
On the contrary, tumour sidedness had not an independent role for median OS $(\operatorname{Exp}(\mathrm{b}): 0.58,95 \% \mathrm{CI}: 0.2-1.3, P=0.20)$, PFS $(\operatorname{Exp}(\mathrm{b}): 0.817,95 \% \mathrm{CI}: 0.4-1.7, P=0.60)$ or ORR $(P=0.95)$.

\section{CONCLUSION}

When it was first proposed the suggestion that primary tumour location might represent a predictive factor for clinical outcome in metastatic CRC patients seemed bound to redefine the therapeutic strategy for these patients. This appeared particularly relevant for anti-EGFR therapy, which demonstrated an impressive activity profile in left-sided primary colorectal tumours and a substantial lack of efficacy in right-sided primary tumours even in presence of an all RAS wild-type mutational status. Nonetheless retrospective observations also suggested that primary tumour sidedness might just represent a surrogate factor substituting in fact for a comprehensive molecular characterisation (Lee et al, 2016b; Tejpar et al, 2016; Venook et al, 2016).

Our analysis confirmed that in RAS/BRAF wild-type colorectal cancer patients, primary tumour location correlated with clinical outcome during irinotecan/cetuximab treatment. Response rate, PFS and OS were in fact reduced in right-sided vs left sided tumours thus indicating that tumour sidedness should be considered among key decision factors in this setting.

As previously observed by other authors and our group, we also confirmed that EGFR GCN and EGFR gene promoter methylation were significantly able to influence clinical outcome in terms of ORR, PFS and OS in these patients (Moroni et al, 2005; Shia et al, 2005; Sartore-Bianchi et al, 2007; Sholl et al, 2007; Cappuzzo et al, 2008; Scartozzi et al, 2009; Scartozzi et al, 2011; Scartozzi et al, 2012). Even more interestingly at multivariate analysis, only EGFR GCN and EGFR gene promoter methylation maintained a significant role in determining clinical results, while primary tumour location failed to independently correlate with ORR, PFS or OS. Our data seemed then to suggest that a thorough knowledge of molecular, genetic and epigenetic tumour characteristics, such as those that we investigated in our study, might overcome the relevance of primary tumour location as a predictive factor during anti-EGFR therapy.

In accordance with our findings, Lee et al showed that on the one hand RSCC was associated with inferior OS and PFS but on the other hand primary tumour sidedness was not a significant factor at multivariate analysis in 198 K-RAS wild-type metastatic

Table 1. Patients characteristics in the whole group and according to primary tumour sidedness, EGFR gene copy number and EGFR gene promoter methylation

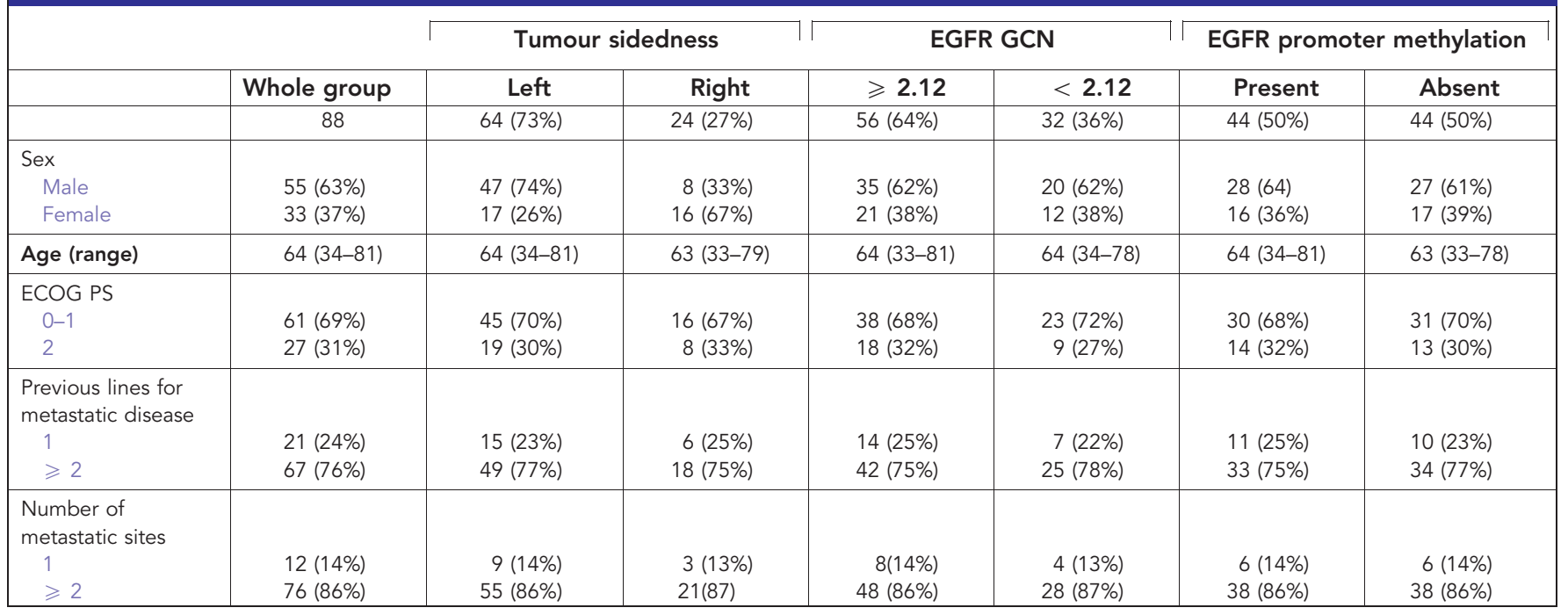



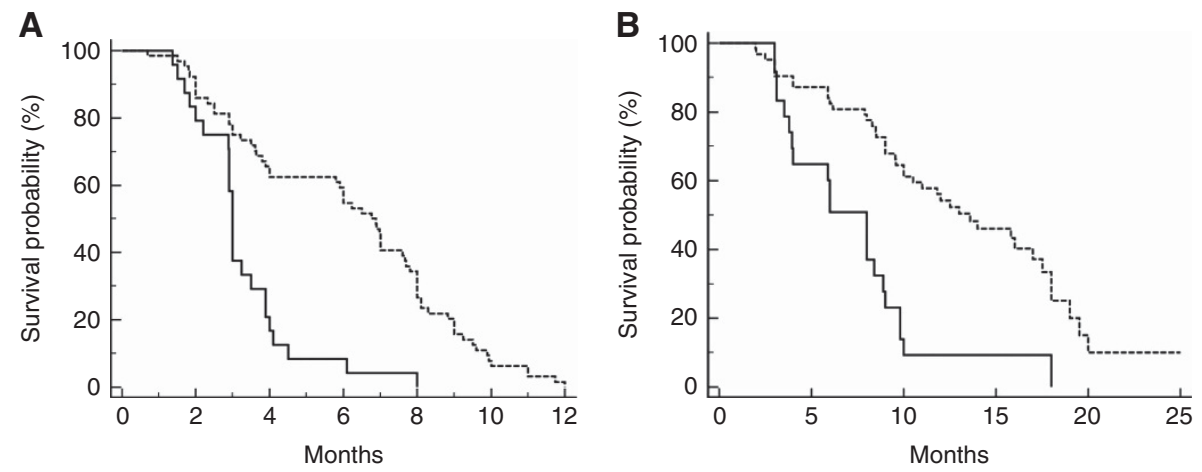

Figure 1. (A) Median progression-free survival (PFS) for patients with right sided (-) or left-sided (- - - -) primary colorectal tumours. Median PFS was 3 vs 6.75 months for patients with right-sided vs left-sided tumours, respectively (HR: 2.74, 95\% Cl: 1.4-5.2, P<0.0001). (B) Median overall survival (OS) for patients with right-sided (-) or left-sided (- - -) primary colorectal tumours. Median OS was 8 vs 13.6 months for patients with right-sided vs left-sided tumours, respectively (HR: 2.83, 95\% Cl: 1.4-5.6, $P<0.0001$ ).
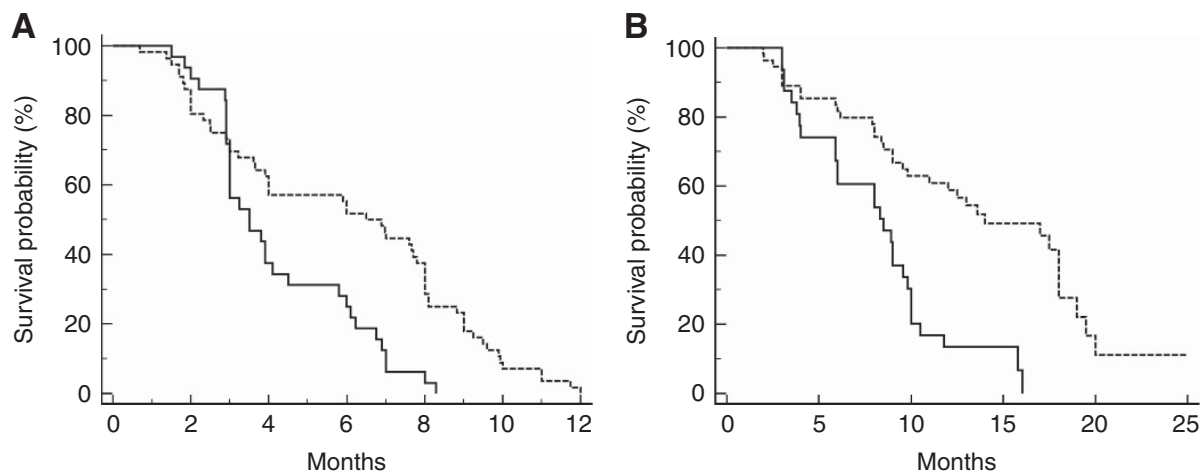

Figure 2. (A) Median progression-free survival (PFS) according to tumour EGFR GCN $<2.12$ (-) or tumour EGFR GCN $\geq 2.12$ (- - - -). Median PFS for patients with tumour EGFR GCN $<2.12$ vs tumour EGFR GCN $\geqslant 2.12$ was 3.5 months vs 6.5 months, respectively (HR: 1.98 , 95\% Cl: $1.2-$ 3.3, $P=0.0006)$. (B) Median overall survival (OS) according to tumour EGFR GCN $<2.12(-)$ or tumour EGFR GCN $\geqslant 2.12(----)$. Median OS was 8.5 months vs 14 months for patients with tumour EGFR GCN $<2.12$ vs tumour EGFR GCN $\geqslant 2.12$ tumour (HR: 2.59, 95\% Cl: 1.4-4.6, P<0.0001).
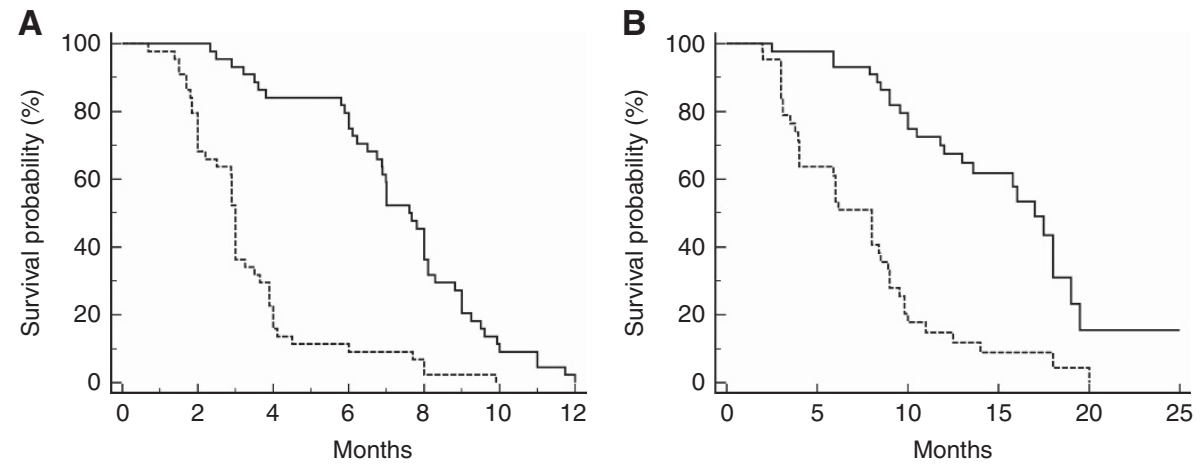

Figure 3. (A) Median progression-free survival (PFS) for patients with EGFR promoter-methylated (- - - -) or EGFR promoter-unmethylated colorectal tumours (-). Median PFS was 3 months vs 7.67 months for patients with EGFR promoter-methylated vs EGFR promoterunmethylated colorectal tumours, respectively (HR: 3.23, 95\% Cl: 2.0-5.3, P<0.0001). (B) Overall survival (OS) for patients with EGFR promotermethylated (- - -) or EGFR promoter-unmethylated tumours (-). Median OS was 8 months vs 17 months for patients with EGFR promotermethylated vs EGFR promoter-unmethylated colorectal tumours, respectively (HR: 3.57, 95\% Cl: 2.1-6.1, $P<0.0001$ ).

colorectal cancer patients receiving anti-EGFR treatment (Lee et al, 2016b). The Authors concluded that tumour biology could explain better than primary tumour anatomical location, the different clinical behaviour during therapy directed against the EGFR. RSCC resulted in fact more frequently associated with BRAF mutations, hypermethylation and a distinct gene expression pattern intriguingly overlapping with the CMS 1 (Guinney et al, 2015; Lee et al, 2016b). In accordance with these findings in our series, EGFR gene promoter methylation was more frequently observed in right-sided primary tumours, whereas EGFR GCN $\geq 2.12$ was more frequent in left-sided primary tumours.

Unlike the study by Lee, the present analysis was not limited to K-RAS but also included H-RAS and BRAF mutational status, thus eliminating potential confounding factors linked to the beyond K-RAS group of mutations with a known predictive and prognostic role. In addition to a broader mutational spectrum, the patients' population examined in our study was truly irinotecan-refractory. Clinical results especially in terms of response rate and PFS were 
Table 2. Clinical outcome results in the whole group and according to primary tumour sidedness, EGFR gene copy number and EGFR gene promoter methylation

\begin{tabular}{|c|c|c|c|c|c|c|c|}
\hline & \multicolumn{2}{|c|}{ Tumour sidedness } & \multicolumn{2}{|c|}{ EGFR GCN } & \multicolumn{2}{|c|}{ EGFR promoter methylation } \\
\hline Number of deaths & $64(72.3 \%)$ & $43(67.1 \%)$ & $21(87.5 \%)$ & $36(64.2 \%)$ & $28(87.5 \%)$ & 38 (86.3\%) & $26(59.0 \%)$ \\
\hline PFS (months) & 4.0 & 6.75 & 3.00 & 6.5 & 3.5 & 3.0 & 7.67 \\
\hline RR & $27.3 \%$ & $35.9 \%$ & $4.2 \%$ & $39.3 \%$ & $6.2 \%$ & $9.1 \%$ & $45.5 \%$ \\
\hline
\end{tabular}

therefore mainly attributable to the activity of cetuximab rather than to chemotherapy.

Unfortunately, although highly suggestive, several limitations prevent from a straightforward application of our findings into the clinical practice. This is in fact a retrospective study with a relatively small sample size that should be mostly considered hypothesis generating. Besides neither EGFR GCN nor EGFR gene promoter methylation is broadly available and standardised.

As long as these observations and those from other authors are not prospectively confirmed primary tumour location should remain a valid surrogate for the molecular profile of colorectal cancer and it should represent an important factor in the decision-making process particularly when an anti-EGFR therapy is anticipated on the basis of both RAS/BRAF mutational status and clinical indication. In patients with RAS/BRAF wild-type LSCRC, chemotherapy in combination with an anti-EGFR monoclonal antibody should then represent a preferred option. Whereas in RAS/BRAF wild-type RSCRC chemotherapy in combination with bevacizumab may be considered the option of choice. Nonetheless, the use of anti-EGFR therapy as a sequential alternative may still be relevant for RAS wild-type RSCRC failing first-line.

In conclusion, it is mandatory to prospectively design sequence trials that are stratified for side and at the same time conduct a parallel translational study to define the role of EGFR GCN and EGFR promoter methylation.

Further studies based also on the influence on outcome of previous and subsequent lines of therapy are needed.

\section{CONFLICT OF INTEREST}

The authors declare no conflict of interest.

\section{REFERENCES}

Cappuzzo F, Finocchiaro G, Rossi E, Jänne PA, Carnaghi C, Calandri C, Bencardino K, Ligorio C, Ciardiello F, Pressiani T, Destro A, Roncalli M, Crino L, Franklin WA, Santoro A, Varella-Garcia M (2008) EGFR FISH assay predicts for response to cetuximab in chemotherapy refractory colorectal cancer patients. Ann Oncol 19: 717-723.

Choi W, Lee J, Lee JY, Lee SM, Kim DW, Kim YJ (2016) Classification of colon cancer patients based on the methylation patterns of promoters. Genomics Inform 14(2): 46-52.

Distler P, Holt PR (1997) Are right- and left-sided colon neoplasms distinct tumors? Dig Dis 15: 302-311.

Ferlay J, Soerjomataram I, Dikshit R, Eser S, Mathers C, Rebelo M, Parkin DM, Forman F, Bray F (2014) Cancer incidence and mortality worldwide: sources, methods and major patterns in GLOBOCAN 2012. Int J Cancer 136(5): E359-E386.
Ferlay J, Soerjomataram I, Ervik M, Dikshit R, Eser S, Mathers C, Rebelo M, Parkin DM, Forman D, Bray F (2013) GLOBOCAN 2012 v1.0, Cancer Incidence and Mortality Worldwide: IARC CancerBase No. 11. International Agency for Research on Cancer: Lyon, France. Available at http://globocan.iarc.fr (accessed on 06/01/2017).

Guinney J, Dienstmann R, Wang X, de Reyniès A, Schlicker A, Soneson C, Marisa L, Roepman P, Nyamundanda G, Angelino P, Bot BM, Morris JS, Simon IM, Gerster S, Fessler E, De Sousa E, Melo F, Missiaglia E, Ramay H, Barras D, Homicsko K, Maru D, Manyam GC, Broom B, Boige V, Perez-Villamil B, Laderas T, Salazar R, Gray JW, Hanahan D, Tabernero J, Bernards R, Friend SH, Laurent-Puig P, Medema JP, Sadanandam A, Wessels L, Delorenzi M, Kopetz S, Vermeulen L, Tejpar S (2015) The consensus molecular subtypes of colorectal cancer. Nat Med 21(11): 1350-1356.

Herman JG, Graff JR, Myöhänen S, Nelkin BD, Baylin SB (1996) Methylationspecific PCR: a novel PCR assay for methylation status of CpG islands. Proc Natl Acad Sci USA 93: 9821-9826.

Hutchins G, Southward K, Handley K, Magill L, Beaumont C, Stahlschmidt J, Richman S, Chambers P, Seymour M, Kerr D, Gray R, Quirke P (2011) Value of mismatch repair, KRAS, and BRAF mutations in predicting recurrence and benefits from chemotherapy in colorectal cancer. J Clin Oncol 29: 1261-1270.

Iacopetta B (2002) Are there two sides to colorectal cancer? Int J Cancer 101: 403-408.

Khambata-Ford S, Garrett CR, Meropol NJ, Basik M, Harbison CT, Wu S, Wong TW, Huang X, Takimoto CH, Godwin AK, Tan BR, Krishnamurthi SS, Burris 3rd HA, Poplin EA, Hidalgo M, Baselga J, Clark EA, Mauro DJ (2007) Expression of epiregulin and amphiregulin and K-ras mutation status predict disease control in metastatic colorectal cancer patients treated with cetuximab. J Clin Oncol 25: 3230-3236.

Lee MG, Advani SA, Morris J, Jiang Z, Manyam GC, Menter D, Broom BM, Eng C, Overman MJ, Maru DM, Hamilton SR, Kopetz S (2016a) Association of primary $\left(1^{\circ}\right)$ site and molecular features with progressionfree survival (PFS) and overall survival (OS) of metastatic colorectal cancer $(\mathrm{mCRC})$ after anti-epidermal growth factor receptor ( $\alpha$ EGFR) therapy. J Clin Oncol 34: 3506.

Lee MS, McGuffey EJ, Morris JS, Manyam G, Baladandayuthapani V, Wei W, Morris VK, Overman MJ, Maru DM, Jiang ZQ, Hamilton SR, Kopetz S (2016b) Association of CpG island methylator phenotype and EREG/ AREG methylation and expression in colorectal cancer. Br J Cancer 114: $1352-1361$.

Loupakis F, Yang D, Yau L, Feng S, Cremolini C, Zhang W, Maus MK, Antoniotti C, Langer C, Scherer SJ, Müller T, Hurwitz HI, Saltz L, Falcone A, Lenz HJ (2015) Primary tumor location as a prognostic factor in metastatic colorectal cancer. J Natl Cancer Inst 107(3): dju427.

Missiaglia E, Jacobs B, D'Ario G, Di Narzo AF, Soneson C, Budinska E, Popovici V, Vecchione L, Gerster S, Yan P, Roth AD, Klingbiel D, Bosman FT, Delorenzi M, Tejpar S (2014) Distal and proximal colon cancers differ in terms of molecular, pathological, and clinical features. Ann Oncol 25: 1995-2001.

Moretto R, Cremolini C, Rossini D, Pietrantonio F, Battaglin F, Mennitto A, Bergamo F, Loupakis F, Marmorino F, Berenato R, Marsico VA, Caporale M, Antoniotti C, Masi G, Salvatore L, Borelli B, Fontanini G, Lonardi S, De Braud F, Falcone A (2016) Location of primary tumor and 
benefit from anti-epidermal growth factor receptor monoclonal antibodies in patients with RAS and BRAF wild-type metastatic colorectal cancer. Oncologist 21: 988-994.

Moroni M, Veronese S, Benvenuti S, Marrapese G, Sartore-Bianchi A, Di Nicolantonio F, Gambacorta M, Siena S, Bardelli A (2005) Gene copy number for epidermal growth factor receptor (EGFR) and clinical response to anti-EGFR treatment in colorectal cancer: a cohort study. Lancet Oncol 5: 279-286.

Nagothu KK, Rishi AK, Jaszewski R, Kucuk O, Majumdar AP (2004) Folic acid- mediated inhibition of serum-induced activation of EGFR promoter in colon cancer cells. Am J Physiol Gastrointestinal Liver Physiol 287: 541-546.

Sartore-Bianchi A, Moroni M, Veronese S, Carnaghi C, Bajetta E, Luppi G, Sobrero A, Barone C, Cascinu S, Colucci G, Cortesi E, Nichelatti M, Gambacorta M, Siena S (2007) Epidermal growth factor receptor gene copy number and clinical outcome of metastatic colorectal cancer treated with panitumumab. J Clin Oncol 25: 3238-3245.

Scartozzi M, Bearzi I, Mandolesi A, Giampieri R, Faloppi L, Galizia E, Loupakis F, Zaniboni A, Zorzi F, Biscotti T, Labianca R, Falcone A, Cascinu S (2011) Epidermal growth factor receptor (EGFR) gene promoter methylation and cetuximab treatment in colorectal cancer patients. $\mathrm{Br} \mathrm{J}$ Cancer 104(11): 1786-1790.

Scartozzi M, Bearzi I, Mandolesi A, Pierantoni C, Loupakis F, Zaniboni A, Negri F, Quadri A, Zorzi F, Galizia E, Berardi R, Biscotti T, Roberto Labianca R, Masi G, Falcone A, Cascinu S (2009) Epidermal Growth Factor Receptor (EGFR) gene copy number (GCN) correlates with clinical activity of irinotecan-cetuximab in K-RAS wild-type colorectal cancer: a fluorescence in situ (FISH) and chromogenic in situ hybridization (CISH) analysis. BMC Cancer 9: 303 .

Scartozzi M, Giampieri R, Maccaroni E, Mandolesi A, Giustini L, Silva R, Zaniboni A, Biscotti T, Biagetti S, Galizia E, Loupakis F, Falcone A, Bearzi I, Cascinu S (2012) Analysis of HER-3, insulin growth factor-1, nuclear factor-kB and epidermal growth factor receptor gene copy number in the prediction of clinical outcome for K-RAS wild-type colorectal cancer patients receiving irinotecan-cetuximab. Ann Oncol 23(7): 1706-1712.

Shia J, Klimstra DS, Li AR, Qin J, Saltz L, Teruya-Feldstein J, Akram M, Chung KY, Yao D, Paty PB, Gerald W, Chen B (2005) Epidermal growth factor receptor expression and gene amplification in colorectal carcinoma: an immunohistochemical and chromogenic in situ hybridization study. Mod Pathol 16: 1-7.

Sholl LM, Iafrate AJ, Chou YP, Wu MT, Goan YG, Su L, Huang YT, Christiani DC, Chirieac LR (2007) Validation of chromogenic in situ hybridization for detection of EGFR copy number amplification in non small cell lung carcinoma. Mod Pathol 20: 1028-1035.

Tejpar S, Stintzing S, Ciardiello F, Tabernero J, Van Cutsem E, Beier F, Esser R, Lenz HJ, Heinemann V (2016) Prognostic and predictive relevance of primary tumor location in patients with RAS wild-type metastatic Colorectal cancer: retrospective analyses of the CRYSTAL and FIRE-3 Trials. JAMA Oncol. e-pub ahead of print 10 October 2016; doi:10.1001/jamaoncol.2016.3797.

Tran B, Kopetz S, Tie J, Gibbs P, Jiang ZQ, Lieu CH, Agarwal A, Maru DM, Sieber O, Desai J (2011) Impact of BRAF mutation and microsatellite instability on the pattern of metastatic spread and prognosis in metastatic colorectal cancer. Cancer 117: 4623-4632.

Van Cutsem E, Cervantes A, Adam R, Sobrero A, Van Krieken JH, Aderka D, Aranda Aguilar E, Bardelli A, Benson A, Bodoky G, Ciardiello F, D’Hoore A, Diaz-Rubio E, Douillard J-Y, Ducreux M, Falcone A, Grothey A, Gruenberger T, Haustermans K, Heinemann V, Hoff P, Köhne C-H, Labianca R, Laurent-Puig P, Ma B, Maughan T, Muro K, Normanno N, Österlund P, Oyen WJG, Papamichael D, Pentheroudakis G, Pfeiffer P, Price TJ, Punt C, Ricke J, Roth A, Salazar R, Scheithauer W, Schmoll HJ, Tabernero J, Taïeb J, Tejpar S, Wasan H, Yoshino T, Zaanan A, Arnold D (2016) ESMO consensus guidelines for the management of patients with metastatic colorectal cancer. Ann Oncol 27: 1386-1422.

Venook A, Niedzwiecki D, Innocenti F, Fruth B, Greene C, O'Neil BH, Shaw J, Atkins J, Horvath LE, Polite B, Meyerhardt JA, O'Reilly EM, Goldberg R, Hochster HS, Blanke CD, Schilsky R, Mayer RJ, Bertagnolli M, Lenz HJ (2016) Impact of primary tumor location on overall survival and progression free survival in patients with metastatic colorectal cancer: analysis of CALGB/SWOG 80405 (Alliance). J Clin Oncol 34: 3504.

This work is published under the standard license to publish agreement. After 12 months the work will become freely available and the license terms will switch to a Creative Commons AttributionNonCommercial-Share Alike 4.0 Unported License. 